\title{
The Value of Programmed Death Ligand 1 Expression in Cancer Patients Treated with Neoadjuvant Chemotherapy
}

Malika Al-Dughaishi, ${ }^{1}$ *Asem Shalaby, ${ }^{2}$ Khawla Al-Ribkhi, ${ }^{1}$ Ammar Boudaka, ${ }^{3}$ Mohamed-Rachid Boulassel, ${ }^{4}$ Jumana Saleh ${ }^{1}$

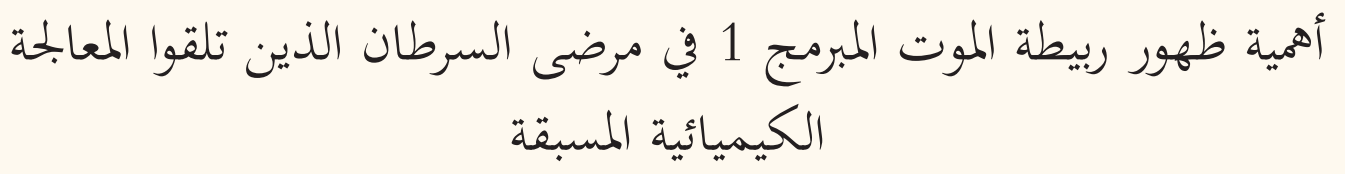

مليكة الدغيشية، عاصم شلبي، خولة الربخية، عمار بودقه، محمد راشد بولعسل، جمانة صالح

ABSTRACT: Programmed death ligand 1 (PD-L1) is an inhibitory molecule expressed by cancer cells to supress T-cell activity and escape anti-tumour immunity. The role of PD-L1 in cancer has been studied extensively as it is considered an important immune checkpoint against immune over-activation through its interaction with Programmed death receptor 1 (PD-1) expressed on activated lymphocytes. PD-L1 expression was found to be enhanced by chemotherapy through different proliferation pathways. However, the predictive and prognostic value for PD-L1 expression in cancer patients treated with neoadjuvant chemotherapy (NAC) is not yet established. This review focused on the potential effects of chemotherapy on PD-L1 expression and the role of PD-L1 as a prognostic and predictive marker in NAC-treated cancer patients. In addition, the potential use of this marker in clinical practice is discussed.

Keywords: Programmed Cell Death 1 Ligand 1; Programmed Cell Death 1 Receptor; Neoadjuvant Therapy; Cancer.

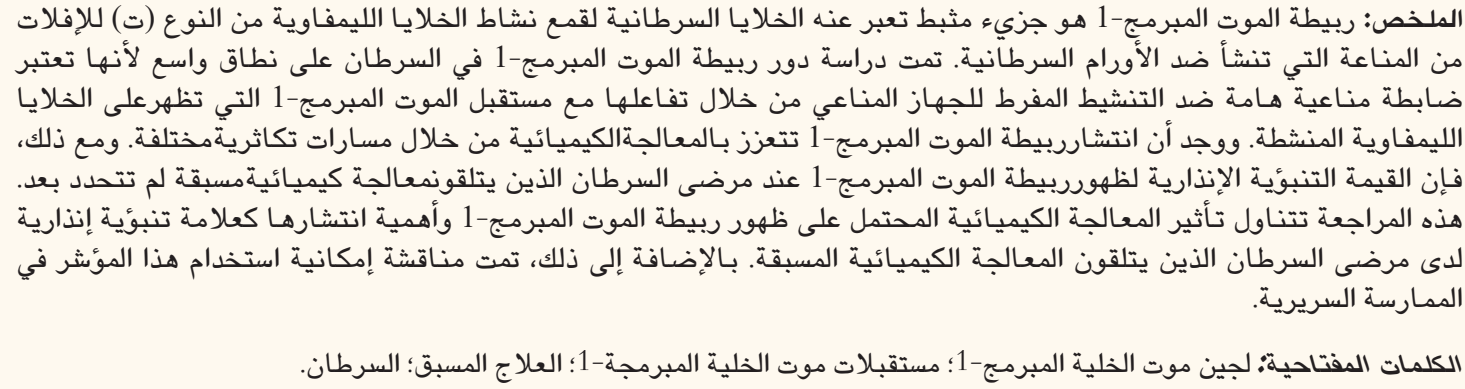

$\mathrm{T}$ HE ROLE OF THE IMMUNE SYSTEM IN CANCER has been studied extensively; immunity in the cancer microenvironment has a role in different phases of cancer development. ${ }^{1}$ Cancer cells usually express abnormal antigens which trigger the immune response to be activated against these cancerous cells. ${ }^{2}$ Tumour-infiltrating lymphocytes (TIL), including cytotoxic T-cells, are usually involved in tumour antigen recognition and anti-tumour immunity to destroy cancer cells during immune-surveillance. ${ }^{1,3}$ Normally, the initiation of the immune response by T-cells is mediated through $\mathrm{T}$-cell receptor (TCR) recognition of the antigen and regulated by inhibitory and stimulatory molecules expressed in these T-cells. The inhibitory molecules are considered as immune checkpoints for self-tolerance (i.e. protection from tissue damage during an immune response and prevention of developing autoimmune diseases). However, the inhibitory effect mediated by these immune checkpoint molecules may also be used as a mechanism for tumour cells to escape the immune response. ${ }^{4}$ One of these immune checkpoints is the programmed death receptor 1 (PD-1)/programmed death ligand 1 (PD-L1) interaction [Figure 1].

PD-1 is a member of cluster of differentiation (CD) 28 family that is expressed in T-cells. ${ }^{5}$ It is also expressed in B-cells, professional antigen presenting cells and natural killer cells. ${ }^{6}$ The PD-L1 was firstly identified by Dong et al. in 1999 and showed that there was an increase in the T-cell proliferation and interleukin (IL)-10 secretion by T-cells stimulated with PD-L1/B7-H1 and low levels of anti-CD3. ${ }^{7}$ In 2000, Freeman et al. showed induction of PD-L1 expression by cytokines as interferon (IFN)- $\gamma$ or other inflammatory stimuli could result in attenuation of 


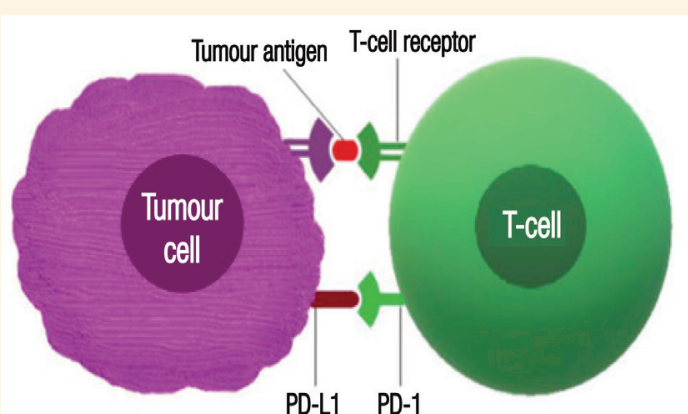

Figure 1: Depiction of the T-cell receptor recognition of the tumour antigen initiating the T-cell response which is regulated by inhibitory molecules expressed in T-cells such as programmed death receptor 1 . However, tumour cells may use this inhibitory effect to escape the immune response.

$P D-L 1=$ programmed death ligand $1 ; P D-1=$ programmed death receptor 1 .

TCR/CD28-mediated T-cell activation which may be significant in the regulation of inflammatory or autoimmune reactions. ${ }^{8}$ PD-L1, also called B7-H1, is a member of B7 molecules which are involved in the regulation of T-cell response either through stimulation or inhibition. ${ }^{9}$ PD-L1 is generally widely expressed in different tissues from different lineages including lymphoid (i.e. immune cells) or non-lymphoid (i.e. epithelial and endothelial) lineages and in cancer cells that originate from these lineages. ${ }^{8,10}$ PD-L1 was found to inhibit proliferation of T-cells and production of cytokines such as IFN- $\gamma$ and IL-2, through interaction with PD-1 expressed on these cells. ${ }^{11}$ In the cancer microenvironment, the interaction of PD-L1 which is expressed in cancer cells and PD-1 which is expressed in T-cells leads to the inhibition of cytotoxic T-cell activity, a decrease in cytokines production by $\mathrm{T}$-cells and induction of T-regulatory cells. Therefore, this interaction protects tumour cells from destruction by cytotoxic T-cells and promotes tumour cell proliferation. ${ }^{1,2,12}$

Many studies chose to investigate the role of PDL1 expression in subjects not treated with neoadjuvant chemotherapy (NAC). For example, a study on triple negative breast cancer (TNBC) patients excluded patients treated with NAC. ${ }^{13}$ Similarly, several studies preferred to include patients who did not receive NAC. ${ }^{14-16}$ This exclusion was partly attributed to the possibility that chemotherapy may contribute to change the expression of specific markers; for example, the expression of progesterone receptor and cellular proliferation marker (Ki-67) in breast cancer was decreased after NAC. ${ }^{17}$

The effect of NAC on PD-L1 expression in cancer patients has not yet been established. Therefore, this review aimed to highlight the effect of chemotherapy on the expression of PD-L1, the potential use of PD-L1 expressed in pre-treated patients as a predictive marker

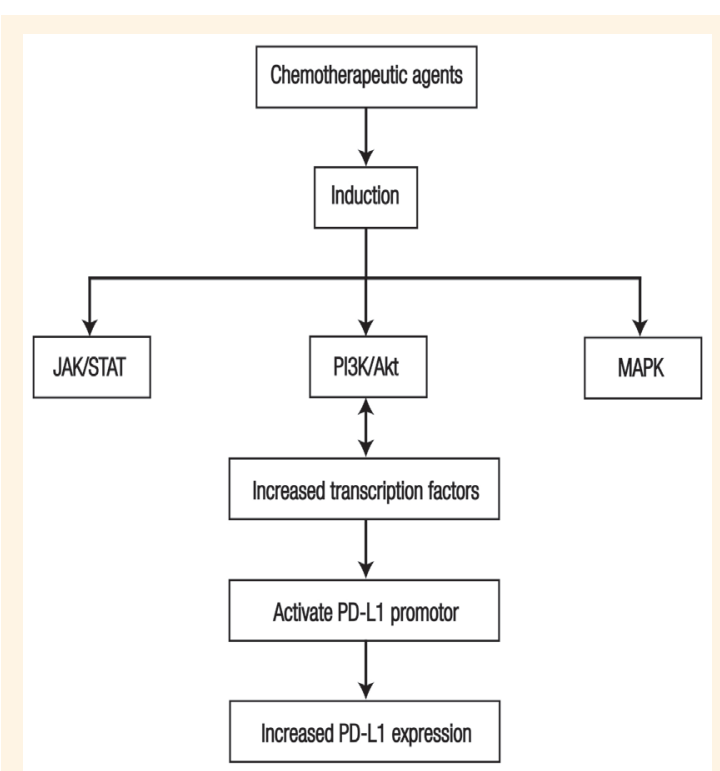

Figure 2: Flowchart showing the different pathways involved in programmed death ligand 1 upregulation induced by chemotherapy treatment.

JAK/STAT = janus-activated kinase//ignal transducers and activators of transcription; $P I 3 K / A k t=$ phosphoinositide -3 kinase $/$ protein kinase $B ; M A P K=$ mitogen-activated protein kinase; $P D-L 1=$ programmed death ligand 1

for the response to chemotherapy and whether it could be used as a prognostic disease marker.

\section{POST-NEOADJUVANT CHEMOTHERAPY CHANGES IN PROGRAMMED DEATH LIGAND 1 EXPRESSION IN DIFFERENT CANCERS}

Immunohistochemical detection of PD-L1 expression can be either increased or decreased by NAC according to the tumour type. It is known that chemotherapy provokes an immune response but, once PD-L1 is overexpressed, this immune response might be diminished. Increased expression of PD-L1 after NAC suggests that the immune response is inhibited which may contribute to chemo-resistance and poor clinical outcome. ${ }^{18}$ Therefore, a combination of chemotherapy and immunotherapy was suggested to overcome this resistance. Conversely, decreased expression of PD-L1 may be associated with a better response to NAC treatment.

\section{Cancers associated with increased Programmed Death Ligand 1 expression}

Increased PD-L1 expression in association with NAC was demonstrated in post-treated specimens of ovarian, bladder and gastrointestinal cancers as well as head and neck squamous cell carcinoma (HNSCC). ${ }^{19-25}$ In epithelial ovarian cancer, 63\% of PD-L1 negative tumours converted to positive PD-L1 tumours after NAC when patients received carboplatin and paclitaxel; the positivity rate in the PD-L1 positive samples increased from $30 \%$ in pre-treated specimens to $53 \%$ in post-treated 


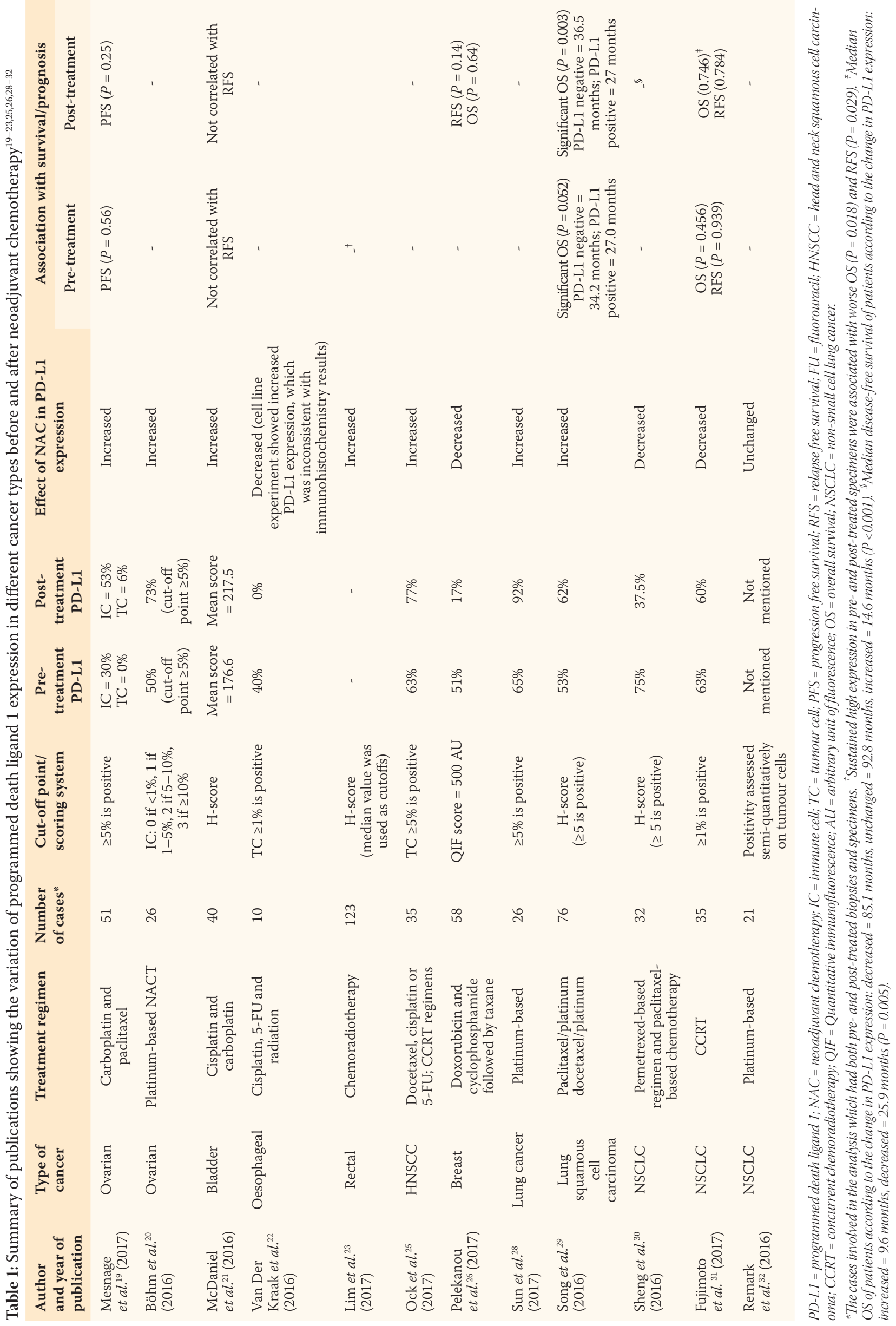


specimens. ${ }^{19}$ This was further supported by findings showing that cisplatin treatment of ovarian cancer cell lines SKOV-3 and OVCAR-3 in vitro induced the expression of PD-L1. ${ }^{19}$ In addition, a high proportion of T-cells expressing PD-L1 were found in biopsies from ovarian high-grade serous carcinoma after NAC. ${ }^{20}$ The expression of PD-L1 was increased in $76 \%$ of patients with urothelial carcinoma treated with cisplatin or carboplatin-based chemotherapy. ${ }^{21}$ Although the increased expression was not observed in post-treated oesophageal carcinoma specimens using immunohistochemistry, 5-fluorouracil induced surface expression of PD-L1 in oesophageal cancer (OE33) and colorectal cancer (HCT 116 p53 $3^{+/+}$and HCT 116 p53-/-) cell lines. ${ }^{22}$ PD-L1 expression and TIL were increased after concurrent chemoradiation therapy (CCRT) in rectal carcinoma. ${ }^{23}$ Notably, sustained high expression of PDL1 in pre- and post-chemoradiotherapy specimens, associated with less increase in TIL, showed poorer overall survival (OS) and disease-free survival (DFS) compared to other patients. ${ }^{23}$

Inhibition of PD-L1 may be beneficial only in some ovarian cancer patients. PD-L1 is expressed significantly more in high grade tumours than in low grade tumours; therefore, a subgroup of advanced disease ovarian cancer patients with high grade tumours, expressing PD-L1, may benefit the most from therapy targeting PD-L1. ${ }^{19}$

In HNSCC, immunohistochemical analysis of 13 negative cases of PD-L1 showed that nine cases (69.2\%) showed increased PD-L1 expression after cisplatin treatment. ${ }^{25}$ Furthermore, patients who received neoadjuvant cisplatin treatment showed more PD-L1 upregulation compared to patients who did not receive cisplatin. ${ }^{25}$ This was supported by findings showing increased PD-L1 expression in HNSCC cell lines (SNU-1066, SNU-1041, SNU-1076, Detroit-562, FaDu and PCI-13) treated with cisplatin alone. ${ }^{25}$

\section{Cancers associated with decreased Programmed Death Ligand 1 expression}

Conversely, PD-L1 was downregulated in breast and squamous oral carcinoma, after treatment with NAC. ${ }^{26,27}$ In the analysis of 58 breast specimens (56\% of patients treated with doxorubicin and cyclophosphamide followed by taxane and the remaining patients received other agents), the positivity rate of PD-L1 expression decreased from $51 \%$ to $17.2 \%$ and 17 cases converted from positive to negative status in spite of the increase of T-cells. ${ }^{26}$ The comparison between NAC-treated and non-treated squamous oral carcinoma specimens showed that most of the treated specimens weakly expressed PD-L1, while non-treated specimens showed either strong or intermediate expression. Similarly, PD-1 expression was significantly less in NAC-treated specimens. ${ }^{27}$

\section{Programmed Death Ligand 1 expression in lung cancer}

In lung cancer, the expression of PD-L1 showed variable results regarding PD-L1 expression after NAC treatment. Sun et al. showed that PD-L1 expression significantly increased in platinum-based-treated specimens. ${ }^{28} \mathrm{PD}-\mathrm{L} 1$ expression also increased in nine patients and decreased in two patients with lung squamous cell carcinoma after NAC; the positivity rate changed from $52.6 \%$ to $61.8 \%{ }^{29}$ Expectedly, expression of PD-L1 was associated with worse DFS compared to the decreased expression. However, PD-L1 expression was not an independent predictor of DFS as determined by multiple regression analysis. $^{30}$

Non-small cell lung cancer samples showed an overall decrease in PD-L1 expression after CCRT. The decreased PD-L1 expression was associated with better survival compared to samples with increased PD-L1 expression (85.1 months versus 14.6 months). ${ }^{31}$ These findings were comparable to another study involving patients with non-small lung carcinoma treated with a pemetrexed-based regimen and paclitaxel-based chemotherapy. ${ }^{30}$ In this study, the positivity rate of PDL1 changed from $75 \%$ to $37.5 \%$ with effect on immune cell infiltration; decreased PD-L1 expression in tumour cells was associated with a better response to NAC. Remark et al's study found that PD-L1 expression in lung cancer was not changed by cisplatin-based NAC treatment indicating that NAC did not contribute to the modification of PD-L1 expression. ${ }^{32}$ Various results from different studies suggest that PD-L1 expression, in response to NAC treatment, may be cancer specific and that different NAC modalities may trigger distinct changes in PD-L1 expression involving different mechanisms.

\section{PROPOSED MECHANISMS OF CHEMO- THERAPY-MEDIATED-PROGRAMMED DEATH LIGAND 1 EXPRESSION CHANGES}

Few studies have explored the mechanisms linked to PD-L1 expression changes associated with NAC. Chemotherapy may be involved in proliferative pathways that can lead to enhanced PD-L1 expression in cancer [Figure 2]. ${ }^{33}$

For instance, PD-L1 expression at the protein and mRNA levels was increased under the influence of 5-fluorouracil, gemcitabine and paclitaxel chemotherapy in pancreatic cancer cell lines (MIA PaCa2, AsPC-1, Pan02). ${ }^{34}$ These three anti-cancer agents increased PD-L1 through Janus-activated kinase/signal transducers and activators of transcription signalling pathways and through phosphorylation by phosphoinositide-3 kinase/protein kinase B (PI3K/Akt) and mitogen-activated protein kinase (MAPK) pathways. ${ }^{34}$ 
In addition, HNSCC cancer cell lines (SNU-1066, SNU-1041, SNU-1076, Detroit-562, FaDu and PCI13) treated with cisplatin showed an increase in PDL1 expression along with up-regulation of extracellular signal-regulated kinase/MAPK. These pathways were found to contribute to chemoresistance. For example, activation of PI3K/Akt pathway was associated with chemotherapy resistance in urothelial carcinoma. ${ }^{35}$ However, targeting the MAPK pathway enhanced the sensitivity of the tumour resistance to cisplatin and helped to overcome this resistance. ${ }^{36}$

Inactivation of programmed cell death (i.e. apoptosis), is essential for cancer development. It was suggested that disabling apoptosis by anti-apoptotic factors may contribute to NAC resistance. For example, a gastric cancer cell line (SGC7901/5-FU) resistant to treatment with 5-fluorouracil was associated with overexpression of PD-L1 compared to a sensitive gastric cell line. A PD-L1 induced resistance was associated with up-regulation of anti-apoptotic proteins, including ATP binding cassette subfamily $C$ member 1 and antiapoptotic factor B-cell lymphoma 2 , that may contribute to the resistance. The blockage of PD-L1 by siRNA was found to overcome this resistance and inhibit proliferation. ${ }^{37}$

A novel mechanism of PD-L1 downregulation of expression by chemotherapy was discovered. ${ }^{38}$ Anthracycline agents, including doxorubicin, were found to decrease the surface expression of PD-L1 in breast cancer cell lines (MDA-MB231 and SKBr3) in a dose and time-dependent manner. However, this effect was correlated with nuclear translocation of PD-L1 and increased nuclear phosphorylated Akt. PD-L1 was also found to have an anti-apoptotic function, in which its blockade by PD-L1 siRNA enhanced the effect of doxorubicin-induced apoptosis and which was PI3K/ Akt pathway dependent. ${ }^{38}$

PROGRAMMED DEATH LIGAND 1 AS A POTENTIAL PREDICTIVE MEASURE FOR THE RESPONSE TO NEOADJUVANT CHEMOTHERAPY

PD-L1 expressed in pre-treated specimen has been investigated to identify patients who would be most likely to respond to NAC. Several studies have found that PD-L1 expression is associated with a pathological complete response (pCR) after NAC in breast cancer. ${ }^{39-42}$ The analysis of 94 biopsies of breast cancer tissue revealed that $\mathrm{pCR}$ was associated with the epithelial and stromal expression of PD-L1. When patients were classified into groups, this association remained significant in hormone-positive and Her2-postive breast cancer. ${ }^{39}$ Similarly, PD-L1 expression in tumour cells was associated with pCR in 54 biopsies of TNBC which remained significant in multivariate analysis. All patients with more than 25\% expression of PDL1 and high TIL achieved pCR. ${ }^{40}$ Expression of PDL1 in tumour cells of Her2-positive breast cancer tumours showed a trend of association with pCR but did not reach significance. ${ }^{41}$ The evaluation of several predictive markers revealed that 20 out of 27 PD-L1positve invasive breast cancer cases showed excellent response to NAC using the Residual Cancer Burden score. ${ }^{41}$ The mechanisms underlying the differential response amongst different molecular types of breast carcinoma may be due to the differing biology of the three types i.e. the tumours may or may not be immunogenic.

Only Baras et al's study of bladder cancer found that PD-L1 was not associated with $\mathrm{pCR} .{ }^{43}$ The expression of PD-L1 in invasive bladder carcinoma showed no significant difference between responders and resistant patients to cisplatin-based NAC. ${ }^{43}$

\section{PROGRAMMED DEATH LIGAND 1 AS A PROGNOSTIC MARKER IN PATIENTS TREATED WITH NEOADJUVANT CHEMO- THERAPY}

The role of PD-L1 as a prognostic marker in patients treated with NAC remains largely unknown. The results of several studies identifying the expression of PD-L1 in residual tumours (i.e. post-treated specimens) have been concordant. It has been reported that high PD-L1 expression in residual tumours was associated with poor clinical outcome in locally-advanced breast cancer after NAC. According to molecular subtypes, the association was more significant in the TNBC subtype. ${ }^{44}$ Patients with high PD-L1 expression and low $\mathrm{CD}^{+}$cells had a poor outcome compared to other groups. ${ }^{44}$ However, some studies found that PD-L1 expression in residual breast tumours was not associated with the clinical outcome. ${ }^{26,41}$ In posttreated bladder cancer, disease-specific survival in PD-L1-positive patients was significantly lower than in PD-L1-negative patients. ${ }^{45}$ In lung cancer, both preand post-treated expression of PD-L1 was associated with worse OS; however, the association was significant and independent in post-treated specimens indicating that PD-L1 status after NAC treatment is a better prognostic marker than PD-L1 status prior to NAC treatment. ${ }^{29}$

\section{Conclusion}

Chemotherapy may cause an increase or decrease in the expression of PD-L1; this outcome seems to be cancer specific as shown by immunohistochemistry 
analysis of pre- and post-treated specimens and cell line experiments. It is possible that PD-L1 is involved in chemoresistance through inhibiting the induction of T-cell activity pathways. Therefore, it may be important to consider combining immunotherapy and chemotherapy to improve the response to the treatment. Current evidence showed that increased PD-L1 expression in post-treated specimens is a poor prognostic marker; although, PD-L1 was shown to predict the response to NAC in breast cancer. Further studies are needed to validate this predictive role of PD-L1 in response to NAC in other cancers except breast cancer. Also, further broader investigations are required to explore the prognostic role of PD-L1.

\section{ACKNOWLEDGEMENT}

The authors would like to thank Dr Elias Said for his input and valuable comments.

\section{References}

1. Kurozumi S, Fujii T, Matsumoto H, Inoue K, Kurosumi M, Horiguchi J, et al. Significance of evaluating tumor-infiltrating lymphocytes (TILs) and programmed cell death-ligand 1 (PD-L1) expression in breast cancer. Med Mol Morphol 2017; 50:185-94. https://doi.org/10.1007/s00795-017-0170-y.

2. Topalian SL, Drake CG, Pardoll DM. Targeting the PD-1/B7H1(PD-L1) pathway to activate anti-tumor immunity. Curr Opin Immunol 2012; 24:207-12. https://doi.org/10.1016/j.coi.2 011.12.009.

3. Dunn GP, Bruce AT, Ikeda H, Old LJ, Schreiber RD. Cancer immunoediting: From immunosurveillance to tumor escape. Nat Immunol 2002; 3:991-8. https://doi.org/10.1038/ni1102-991.

4. Pardoll DM. The blockade of immune checkpoints in cancer immunotherapy. Nat Rev Cancer 2012; 12:252-64. https://doi. org $/ 10.1038 /$ nrc3239

5. Guan J, Lim KS, Mekhail T, Chang CC. Programmed Death Ligand-1 (PD-L1) expression in the Programmed Death Receptor-1 (PD-1)/PD-L1 blockade: A key player against various cancers. Arch Pathol Lab Med 2017; 141:851-61. https://doi. org/10.5858/arpa.2016-0361-RA.

6. Bio-Rad Laboratories, Inc. The Role of Immune Checkpoints in Immunity and Cancer. From: https://www.bio-rad-antibodies. com/immune-checkpoint-minireview.html Accessed: Sep 2019.

7. Dong H, Zhu G, Tamada K, Chen L. B7-H1, a third member of the B7 family, co-stimulates T-cell proliferation and interleukin-10 secretion. Nat Med 1999; 5:1365-9. https://doi.org/10.1 038/70932.

8. Freeman GJ, Long AJ, Iwai Y, Bourque K, Chernova T, Nishimura H, et al. Engagement of the PD-1 immunoinhibitory receptor by a novel B7 family member leads to negative regulation of lymphocyte activation. J Exp Med 2000; 192:1027-34. https://doi.org/10.1084/jem.192.7.1027.

9. Ni L, Dong C. New B7 family checkpoints in human cancers. Mol Cancer Ther 2017; 16:1203-11. https://doi.org/10.1158/15 35-7163.MCT-16-0761.

10. Gibbons Johnson RM, Dong H. Functional expression of Programmed Death-Ligand 1 (B7-H1) by immune cells and tumor cells. Front Immunol 2017; 8:961. https://doi.org/10.33 89/fimmu.2017.00961.
11. Freeman GJ, Long AJ, Iwai Y, Bourque K, Chernova T, Nishimura H, et al. Engagement of the PD-1 immunoinhibitory receptor by a novel B7 family member leads to negative regulation of lymphocyte activation. J Exp Med 2000; 192:1027-34. https://doi.org/10.1084/jem.192.7.1027.

12. Bertucci F, Gonçalves A. Immunotherapy in breast cancer: The emerging role of PD-1 and PD-L1. Curr Oncol Rep 2017; 19:64. https://doi.org/10.1007/s11912-017-0627-0.

13. Mori H, Kubo M, Yamaguchi R, Nishimura R, Osako T, Arima N, et al. The combination of PD-L1 expression and decreased tumor-infiltrating lymphocytes is associated with a poor prognosis in triple-negative breast cancer. Oncotarget 2017; 8:15584-92. https://doi.org/10.18632/oncotarget.14698.

14. Baptista MZ, Sarian LO, Derchain SF, Pinto GA, Vassallo J. Prognostic significance of PD-L1 and PD-L2 in breast cancer. Hum Pathol 2016; 47:78-84. https://doi.org/10.1016/j.humpa th.2015.09.006

15. Wang ZQ, Milne K, Derocher H, Webb JR, Nelson BH, Watson PH. PD-L1 and intratumoral immune response in breast cancer. Oncotarget 2017; 8:51641-51. https://doi.org/10.18632/onco target. 18305 .

16. Kim A, Lee SJ, Kim YK, Park WY, Park DY, Kim JY, et al. Programmed Death-Ligand 1 (PD-L1) expression in tumour cell and tumour infiltrating lymphocytes of HER2-positive breast cancer and its prognostic value. Sci Rep 2017; 7:11671. https://doi.org/10.1038/s41598-017-11905-7.

17. Lee H, Ko H, Seol H, Noh DY, Han W, Kim TY, et al. Expression of immunohistochemical markers before and after neoadjuvant chemotherapy in breast carcinoma, and their use as predictors of response. J Breast Cancer 2013; 16:395-403. https://doi.org/10.4 048/jbc.2013.16.4.395.

18. Liu Q, Gong W, Cheng Z, Jiang H, Cao F, Li X. Counteracting immunosuppressive cofactor B7-H1 may enhance the effects of chemotherapy in cancer patients. Immunotherapy 2013; 5:557-9. https://doi.org/10.2217/imt.13.38.

19. Mesnage SJL, Auguste A, Genestie C, Dunant A, Pain E, Drusch F, et al. Neoadjuvant Chemotherapy (NACT) increases immune infiltration and Programmed Death-Ligand 1 (PD-L1) expression in Epithelial Ovarian Cancer (EOC). Ann Oncol 2017; 28:651-7. https://doi.org/10.1093/annonc/mdw625.

20. Böhm S, Montfort A, Pearce OM, Topping J, Chakravarty P, Everitt GL, et al. Neoadjuvant chemotherapy modulates the immune microenvironment in metastases of tubo-ovarian highgrade serous carcinoma. Clin Cancer Res 2016; 22:3025-36. https://doi.org/10.1158/1078-0432.CCR-15-2657.

21. McDaniel AS, Alva A, Zhan T, Xiao H, Cao X, Gursky A, et al. Expression of PD-L1 (B7-H1) before and after neoadjuvant chemotherapy in urothelial carcinoma. Eur Urol Focus 2016; 1:265-8. https://doi.org/10.1016/j.euf.2015.03.004.

22. Van Der Kraak L, Goel G, Ramanan K, Kaltenmeier C, Zhang L, Normolle DP, et al. 5-fluorouracil upregulates cell surface B7-H1 (PD-L1) expression in gastrointestinal cancers. J Immunother Cancer 2016; 4:65. https://doi.org/10.1186/s40425-016-0163-8.

23. Lim YJ, Koh J, Kim S, Jeon SR, Chie EK, Kim K, et al. Chemoradiation-induced alteration of programmed death-ligand 1 and CD8+ tumor-infiltrating lymphocytes identified patients with poor prognosis in rectal cancer: A matched comparison analysis. Int J Radiat Oncol Biol Phys 2017; 99:1216-24. https://doi.org/10.1016/j.ijrobp.2017.07.004.

24. Goel G, Ramanan K, Kaltenmeier C, Zhang L, Freeman GJ, Normolle DP, et al. Effect of 5-fluorouracil on membranous PDL1 expression in colon cancer cells. J Clin Oncol 2016; 34:592. https://doi.org/10.1200/jco.2016.34.4_suppl.592.

25. Ock CY, Kim S, Keam B, Kim S, Ahn YO, Chung EJ, et al. Changes in programmed death-ligand 1 expression during cisplatin treatment in patients with head and neck squamous cell carcinoma. Oncotarget 2017; 8:97920-7. https://doi.org/10.186 32/oncotarget.18542. 
26. Pelekanou V, Carvajal-Hausdorf DE, Altan M, Wasserman B, Carvajal-Hausdorf $\mathrm{C}$, Wimberly $\mathrm{H}$, et al. Effect of neoadjuvant chemotherapy on tumor-infiltrating lymphocytes and PD-L1 expression in breast cancer and its clinical significance. Breast Cancer Res 2017; 19:91. https://doi.org/10.1186/s13058-017-0884-8.

27. Takakura H, Domae S, Ono T, Sasaki A. The immunological impact of chemotherapy on the tumor microenvironment of oral squamous cell carcinoma. Acta Med Okayama 2017; 71:219-26. https://doi.org/10.18926/AMO/55204.

28. Sun W, Ma K, Wang X, Lui Y, He H, Guo Y. JCES 01.16 Altered expression of Programmed Death-1 Receptor (PD-1) and its ligand PD-L1, PD-L2 after neo-adjuvant chemotherapy in lung cancer. J Thorac Oncol 2017; 12:S1736. https://doi.org/10.10 16/j.jtho.2017.09.303.

29. Song Z, Yu X, Zhang Y. Altered expression of programmed death-ligand 1 after neoadjuvant chemotherapy in patients with lung squamous cell carcinoma. Lung Cancer 2016; 99:166-171. https://doi.org/10.1016/j.lungcan.2016.07.013.

30. Sheng J, Fang W, Yu J, Chen N, Zhan J, Ma Y, et al. Expression of programmed death ligand-1 on tumor cells varies pre and post chemotherapy in non-small cell lung cancer. Sci Rep 2016; 6:20090. https://doi.org/10.1038/srep20090.

31. Fujimoto D, Uehara K, Sato Y, Sakanoue I, Ito M, Teraoka S, et al. Alteration of PD-L1 expression and its prognostic impact after concurrent chemoradiation therapy in non-small cell lung cancer patients. Sci Rep 2017; 7:11373. https://doi.org/10.1038/ s41598-017-11949-9.

32. Remark R, Lupo A, Alifano M, Biton J, Ouakrim H, Stefani A, et al. Immune contexture and histological response after neoadjuvant chemotherapy predict clinical outcome of lung cancer patients. Oncoimmunology 2016; 5:e1255394. https://doi.org/10.1 080/2162402X.2016.1255394

33. Ritprajak P, Azuma M. Intrinsic and extrinsic control of expression of the immunoregulatory molecule PD-L1 in epithelia cells and squamous cell carcinoma. Oral Oncol 2015; 51:221-8. https://doi.org/10.1016/j.oraloncology.2014.11.014

34. Doi T, Ishikawa T, Okayama T, Oka K, Mizushima K, Yasuda T, et al. The JAK/STAT pathway is involved in the upregulation of PD-L1 expression in pancreatic cancer cell lines. Oncol Rep 2017; 37:1545-54. https://doi.org/10.3892/or.2017.5399.

35. Liu ST, Hui G, Mathis C, Chamie K, Pantuck AJ, Drakaki A, et al The current status and future role of the phosphoinositide 3 kinase/AKT signaling pathway in urothelial cancer: An old pathway in the new immunotherapy era. Clin Genitourin Cancer 2018; 16:e269-76. https://doi.org/10.1016/j.clgc.2017.10.011.
36. Achkar IW, Abdulrahman N, Al-Sulaiti H, Joseph JM, Uddin S, Mraiche F. Cisplatin based therapy: The role of the mitogen activated protein kinase signaling pathway. J Transl Med 2018; 16:96. https://doi.org/10.1186/s12967-018-1471-1.

37. Liu N, Lv J, Qi W, Sun L, Guo J, Khao S, et al. Programmed death 1 induces cell chemoresistance to 5-fluorouracil in gastric cancer cell lines. Transl Cancer Res 2016; 5:781-8. https://doi.org/10.21037/tcr.2016.12.01.

38. Ghebeh H, Lehe C, Barhoush E, Al-Romaih K, Tulbah A, Al-Alwan M, et al. Doxorubicin downregulates cell surface B7$\mathrm{H} 1$ expression and upregulates its nuclear expression in breast cancer cells: Role of B7-H1 as an anti-apoptotic molecule. Breast Cancer Res; 12:R48. https://doi.org/10.1186/bcr2605.

39. Wimberly H, Brown JR, Schalper K, Haack H, Silver MR, Nixon C, et al. PD-L1 expression correlates with tumorinfiltrating lymphocytes and response to neoadjuvant chemotherapy in breast cancer. Cancer Immunol Res 2015; 3:326-32. https://doi.org/10.1158/2326-6066.CIR-14-0133.

40. Cerbelli B, Pernazza A, Botticelli A, Fortunato L, Monti M, Sciattella P, et al. PD-L1 expression in TNBC: A predictive biomarker of response to neoadjuvant chemotherapy? Biomed Res Int 2017; 2017:1750925. https://doi.org/10.1136/esmoopen-20 16-000150.

41. Kitano A, Ono M, Yoshida M, Noguchi E, Shimomura A, Shimoi T, et al. Tumour-infiltrating lymphocytes are correlated with higher expression levels of PD-1 and PD-L1 in early breast cancer. ESMO Open 2017; 2:e000150. https://doi.org/10.1136/ esmoopen-2016-000150

42. McLemore LE, Janakiram M, Albanese J, Shapiro N, Lo Y, Zang X, et al. An immunoscore using PD-L1, CD68, and Tumor-Infiltrating Lymphocytes (TILs) to predict response to neoadjuvant chemotherapy in invasive breast cancer. Appl Immunohistochem Mol Morphol 2018; 26:611-19. https://doi. org/10.1097/PAI.0000000000000485.

43. Baras AS, Drake C, Liu JJ, Gandhi N, Kates M, Hoque MO, et al. The ratio of CD8 to Treg tumor-infiltrating lymphocytes is associated with response to cisplatin-based neoadjuvant chemotherapy in patients with muscle invasive urothelial carcinoma of the bladder. Oncoimmunology 2016; 5:e1134412. https://doi. org/10.1080/2162402X.2015.1134412.

44. Chen S, Wang RX, Liu Y, Yang WT, Shao ZM. PD-L1 expression of the residual tumor serves as a prognostic marker in local advanced breast cancer after neoadjuvant chemotherapy. Int J Cancer 2017; 140:1384-95. https://doi.org/10.1002/ijc.30552.

45. Noro D, Hatakeyama S, Yoneyama T, Hashimoto Y, Koie T, Kawaguchi T, et al. Post-chemotherapy PD-L1 expression correlates with clinical outcomes in Japanese bladder cancer patients treated with total cystectomy. Med Oncol 2017; 34:117. https://doi.org/10.1007/s12032-017-0977-3. 